Journal of Organometallic Chemistry, 368 (1989) 67-76

Elsevier Sequoia S.A., Lausanne - Printed in The Netherlands

JOM 09782

\title{
Komplexchemie perhalogenierter Cyclopentadiene und Alkine
}

\section{$V^{*}$. Darstellung weiterer funktioneller Derivate von $\left(\mathrm{C}_{5} \mathrm{Cl}_{4} \mathrm{R}\right) \mathrm{Mn}(\mathrm{CO})_{3}$ und $\left(\mathrm{C}_{5} \mathrm{Cl}_{4} \mathrm{R}\right) \mathrm{Rh}(\mathrm{COD})$. Kristallstruktur von $\left(\mathrm{C}_{5} \mathrm{Cl}_{4} \mathrm{CONH}_{2}\right) \mathrm{Mn}(\mathrm{CO})_{3}$}

\author{
Karlheinz Sünkel * und Doris Steiner \\ Inst. für anorg. Chemie der Universität, D-8000 München 2, Meiserstr. $I$ (B.R.D.) \\ (Eingegangen den 21. Dezember 1988)
}

\begin{abstract}
Starting from $\left(\mathrm{C}_{5} \mathrm{Cl}_{4} \mathrm{Li}\right) \mathrm{Mn}(\mathrm{CO})_{3}$, generated in situ, the functional derivatives $\left(\mathrm{C}_{5} \mathrm{Cl}_{4} \mathrm{R}\right) \mathrm{Mn}(\mathrm{CO})_{3}$ with $\mathrm{R}=\mathrm{SnMe}_{3}, \mathrm{PPh}_{2}, \mathrm{SePh},\left(\mathrm{SCl}_{5} \mathrm{Cl}_{4}\right) \mathrm{Mn}(\mathrm{CO})_{3}, \mathrm{CHO}, \mathrm{COCl}$, $\mathrm{CONH}_{2}, \mathrm{CN}$, NCO, and $\mathrm{NH}_{2}$ can be obtained. A series of compounds $\left(\mathrm{C}_{5} \mathrm{Cl}_{4} \mathrm{R}\right) \mathrm{Rh}(1,5-\mathrm{COD})$ with $\mathrm{R}=\mathrm{H}, \mathrm{Me}, \mathrm{SiMe}_{3}, \mathrm{SiMe}_{2} \mathrm{H}$ and $\mathrm{SnMe}_{3}$ can be prepared from $\left(\mathrm{C}_{5} \mathrm{Cl}_{5}\right) \mathrm{Rh}(1,5-\mathrm{COD})$ via the lithio derivative. The crystal structure of $\left(\mathrm{C}_{5} \mathrm{Cl}_{4} \mathrm{CONH}_{2}\right) \mathrm{Mn}(\mathrm{CO})_{3}$ has been determined.
\end{abstract}

\section{Zusammenfassung}

Aus dem in situ erzeugten $\left(\mathrm{C}_{5} \mathrm{Cl}_{4} \mathrm{Li}\right) \mathrm{Mn}(\mathrm{CO})_{3}$ lassen sich die funktionalisierten Derivate $\left(\mathrm{C}_{5} \mathrm{Cl}_{4} \mathrm{R}\right) \mathrm{Mn}(\mathrm{CO})_{3}$ mit $\mathrm{R}=\mathrm{SnMe}_{3}, \mathrm{PPh}_{2}, \mathrm{SePh},\left(\mathrm{SC}_{5} \mathrm{Cl}_{4}\right) \mathrm{Mn}(\mathrm{CO})_{3}, \mathrm{CHO}$, $\mathrm{COCl}, \mathrm{CONH}_{2}, \mathrm{CN}, \mathrm{NCO}$ und $\mathrm{NH}_{2}$ gewinnen. Auch aus $\left(\mathrm{C}_{5} \mathrm{Cl}_{5}\right) \mathrm{Rh}(1,5-\mathrm{COD})$ wird über das in situ erzeugte Lithioderivat eine Reihe von Verbindungen $\left(\mathrm{C}_{5} \mathrm{Cl}_{4} \mathrm{R}\right) \mathrm{Rh}(\mathrm{COD})$ mit $\mathrm{R}=\mathrm{H}, \mathrm{Me}, \mathrm{SiMe}_{3}, \mathrm{SiMe}_{2} \mathrm{H}$, und $\mathrm{SnMe}_{3}$ erhalten. Die Struktur von $\left(\mathrm{C}_{5} \mathrm{Cl}_{4} \mathrm{CONH}_{2}\right) \mathrm{Mn}(\mathrm{CO})_{3}$ wird kristallographisch bestimmt.

\section{Einleitung}

Der nachträgliche Einbau mehrerer funktioneller Gruppen an bereits $\pi$ koordinierte Cyclopentadienylringe ist mit wenigen Ausnahmen (Friedel-CraftsAcylierung, Sulfonierung) schwierig [2], da Reaktionen an anderen Liganden oder Abspaltung des Cyclopentadienylsystems beobachtet werden. Andererseits führt die

\footnotetext{
* IV. Mitteilung siehe Ref. 1.
} 
Reaktion eines bereits funktionalisierten Cyclopentadiens mit einer geeignet erscheinenden Metallverbindung oft nicht zum $\pi$-Komplex. Ein schonender Reaktionsweg, der auch die Einführung sonst nur schwierig zugänglicher funktioneller Gruppen ermöglicht und auch das Potential zur Mehrfachfunktionalisierung beinhaltet, ist die Lithiierung von perhalogenierten Cyclopentadienylkomplexen mit anschließender Umsetzung mit Elektrophilen [3]. Hier berichten wir über weitere Derivate von $\left(\mathrm{C}_{5} \mathrm{Cl}_{4} \mathrm{R}\right) \mathrm{Mn}(\mathrm{CO})_{3}$ und $\left(\mathrm{C}_{5} \mathrm{Cl}_{4} \mathrm{R}\right) \mathrm{Rh}(\mathrm{COD})$.

\section{Ergebnisse und Diskussion}

Wie wir bereits früher beschrieben haben, läßt sich aus $\left(\mathrm{C}_{5} \mathrm{Cl}_{4} \mathrm{Br}\right) \mathrm{Mn}(\mathrm{CO})_{3}(1 \mathrm{a})$ und Butyllithium das überaus reaktive 1b erhalten [3a]. Mit $\mathrm{SnMe}_{3} \mathrm{Cl}, \mathrm{PPh}_{2} \mathrm{Cl}$, $\mathrm{Ph}_{2} \mathrm{Se}_{2}, \mathrm{SCl}_{2}$ und Dimethylformamid reagiert $1 \mathrm{~b}$ zu $2 \mathrm{a}-2 \mathrm{e}$.<smiles>[R][C@H](Cl)c1c(Cl)c(Cl)c(Cl)c([N+]([O-])([O-])Cl)c1Cl</smiles>

ib<smiles>[R]c1c(Cl)c(Cl)c(N=[W])c(Cl)c1Cl</smiles>

2

$$
\begin{array}{llll}
\text { a } & R=\mathrm{SnMe}_{3} \text { e } \mathrm{CHO} \\
\text { b } & \mathrm{PPh}_{2} & \mathrm{NH}_{2} \\
\text { c } & \mathrm{SePh}_{\mathrm{g} \mathrm{CN}} \\
\text { d } & \left(\mathrm{SC}_{5} \mathrm{Cl}_{4}\right) \mathrm{Mn}\left(\mathrm{CO}_{3}\right.
\end{array}
$$

Das Stannylderivat 2a hydrolysiert sehr leicht zur entsprechenden $\mathrm{H}-\mathrm{Verbindung}$. Das Phosphan 2b wird in Lösung langsam zum Phosphanoxid $\left(\mathrm{C}_{5} \mathrm{Cl}_{4} \mathrm{PPh}_{2} \mathrm{O}\right)$ $\mathrm{Mn}(\mathrm{CO})_{3}\left[4^{*}\right]$ durch eindiffundierende Luft oxidiert, und ist auch sonst recht instabil. Die zu dem von uns schon früher beschriebenen Phenylthioderivat [3a] analoge Selenverbindung $2 c$ läßt sich ebenso wie diese erneut lithiieren und mehrfach selenieren.

Ungewöhnlich glatt verläuft die Umsetzung mit $\mathrm{SCl}_{2}$ zu dem symmetrischen zweikernigen Sulfid 2d. Dies ist das Octachloro-analoge des früher beschriebenen, auf andere Weise erhaltenen $\left[(\mathrm{OC})_{3} \mathrm{Mn}\left(\mathrm{C}_{5} \mathrm{H}_{4}\right)\right]_{2} \mathrm{~S}$ [5]. Der Versuch, durch elektrophile Aminierung von $1 \mathrm{~b}$ mitels $\mathrm{MesSO}_{2} \mathrm{ON}\left(\mathrm{SiMe}_{3}\right)_{2} \quad(\mathrm{Mes}=2,4,6-$ trimethylphenyl) [6] zum $\mathrm{N}\left(\mathrm{SiMe}_{3}\right)_{2}$-derivat zu gelangen, schlug fehl. Auf einem Umweg konnte jedoch das $\mathrm{NH}_{2}$-Analoge $2 \mathrm{f}$ erhalten werden.

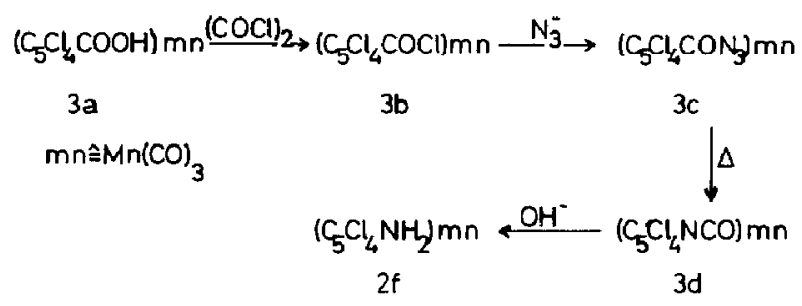

\footnotetext{
* Die Literaturnummer mit einem Sternchen deutet eine Bermerkung in der Literaturliste an.
} 


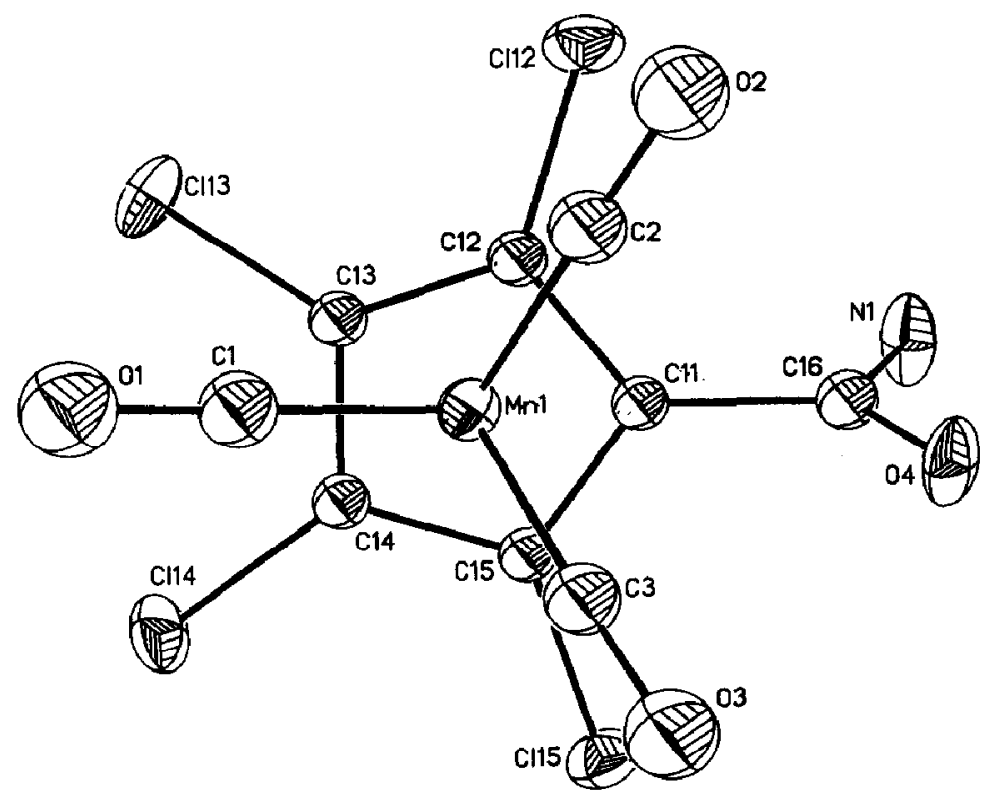

Fig. 1. Struktur von 3e.

Halogenferrocene gehen mit CuCN die Rosenmund-von Braun-Reaktion zum Cyanoferrocen ein [7]. $\left(\mathrm{C}_{5} \mathrm{Cl}_{4} \mathrm{Br}\right) \mathrm{Mn}(\mathrm{CO})_{3}$ und $\mathrm{CuCN}$ ergeben in Pyridin jedoch nur Kupferkomplexe (darunter $\mathrm{CuCl}_{2} \mathrm{py}_{2}, \mathrm{CuBr}_{2} \mathrm{py}_{2}$ und $\left[\mathrm{CuCl}(\mathrm{py})_{2} \mathrm{NCCu}-\right.$ (py) $\left.{ }_{2}(\mathrm{CN})\right]_{x}\left[8^{*}\right]$ als charakterisierbare Produkte. Ausgehend von 3b läßt sich über das Amid 3e jedoch auch das Nitril $2 \mathrm{~g}$ erhalten.

$3 \mathrm{~b}+\mathrm{NH}_{3} \rightarrow\left(\mathrm{C}_{5} \mathrm{Cl}_{4} \mathrm{CONH}_{2}\right) \mathrm{Mn}(\mathrm{CO})_{3} \stackrel{\mathrm{POCl}_{3}}{\longrightarrow}\left(\mathrm{C}_{5} \mathrm{Cl}_{4} \mathrm{CN}\right) \mathrm{Mn}(\mathrm{CO})_{3}$

Die Reaktion von 3e mit Tosylchlorid/Pyridin liefert nicht wie viele "organische" Amide [9] das Nitril 2g, sondern nur ein Strukturisomeres von 3e. Die Feststoff-IRSpektren beider Modifikationen unterscheiden sich deutlich, während Lösungs-IRund NMR-Spektren identisch sind. Vermutlich unterscheiden sich beide Formen in der Orientierung der $\mathrm{CONH}_{2}$-Ebene bezüglich der Cyclopentadienylring-Ebene. Von der 2. Form von 3e konnte die Kristallstruktur bestimmt werden (Fig. 1).

Atomkoordinaten sind Tab. 1, wichtige Bindungslängen und -winkel Tab. 2 zu entnehmen. Die Abweichungen von der Planarität im Fünfring sind gering, es ist jedoch eine gewisse Faltung entlang der Achse C12-C15 festzustellen [10*]. Die $\mathrm{C}-\mathrm{C}$-Bindungslängen bewegen sich im üblichen Rahmen, und auch die $\mathrm{C}-\mathrm{Cl}$ Abstände sind normal. Auffällig an der Struktur ist der Winkel von $114.9^{\circ}$ zwischen den Ebenen des Fünfrings und der Amidgruppe. Eine Wechselwirkung zwischen diesen beiden $\pi$-Systemen ist damit mehr oder weniger ausgeschlossen. Offensichtlich ist der sterische Einfluß der Chlor-Substituenten zu groß, um eine koplanare Einstellung zuzulassen.

An perhalogenierten Cyclopentadienylkomplexen ist neben dem bisher beschriebenen Cymantrenderivat noch $\left(\mathrm{C}_{5} \mathrm{Cl}_{5}\right) \mathrm{Rh}(\mathrm{COD}) \quad(4 a), \quad(\mathrm{COD}=1,5-$ cyclooctadien) relativ einfach zugänglich [11]. Auch dieses ergibt mit Butyllithium 
Tabelle 1

Atomkoordinaten $\left(\times 10^{4}\right)$ und isotrope Thermalparameter $\left(\mathrm{pm}^{2} \times 10^{-1}\right)$ von $3 \mathrm{e}$

\begin{tabular}{lrrrl}
\hline Atom & \multicolumn{1}{l}{$y$} & \multicolumn{1}{l}{$\boldsymbol{z}$} & $U$ \\
\hline $\mathrm{Mn}(1)$ & $2729(1)$ & $1555(1)$ & $2182(1)$ & $42(1)^{a}$ \\
$\mathrm{C}(11)$ & $2199(5)$ & $645(4)$ & $2609(9)$ & $42(2)$ \\
$\mathrm{C}(12)$ & $2225(6)$ & $1108(4)$ & $3762(9)$ & $45(2)$ \\
$\mathrm{C}(13)$ & $3160(6)$ & $1314(4)$ & $4548(9)$ & $45(2)$ \\
$\mathrm{C}(14)$ & $3743(5)$ & $983(4)$ & $3963(9)$ & $43(2)$ \\
$\mathrm{C}(15)$ & $3155(5)$ & $560(4)$ & $2782(9)$ & $43(2)$ \\
$\mathrm{C}(16)$ & $1342(6)$ & $291(4)$ & $1477(10)$ & $50(2)$ \\
O(4) & $1088(4)$ & $387(3)$ & $86(6)$ & $72(3)^{a}$ \\
$\mathrm{~N}(1)$ & $916(6)$ & $-116(4)$ & $2048(8)$ & $84(4)^{a}$ \\
$\mathrm{Cl}(12)$ & $1271(2)$ & $1339(1)$ & $4159(3)$ & $72(1)^{a}$ \\
$\mathrm{Cl}(13)$ & $3561(2)$ & $1815(1)$ & $6131(3)$ & $72(1)^{a}$ \\
$\mathrm{Cl}(14)$ & $4960(2)$ & $1001(1)$ & $4698(3)$ & $73(1)^{a}$ \\
$\mathrm{Cl}(15)$ & $3537(2)$ & $-5(1)$ & $1833(3)$ & $66(1)^{a}$ \\
$\mathrm{C}(1)$ & $3456(7)$ & $2265(5)$ & $2537(11)$ & $65(3)$ \\
O(1) & $3960(5)$ & $2723(4)$ & $2838(9)$ & $95(2)$ \\
$\mathrm{C}(2)$ & $1669(7)$ & $2023(5)$ & $1341(11)$ & $61(2)$ \\
O(2) & $942(5)$ & $2300(4)$ & $803(8)$ & $88(2)$ \\
$\mathrm{C}(3)$ & $2760(6)$ & $1433(4)$ & $363(10)$ & $59(2)$ \\
O(3) & $2761(5)$ & $1311(4)$ & $-833(8)$ & $87(2)$ \\
\hline
\end{tabular}

a Äquivalente isotrope $U$ berechnet als ein Drittel der Spur des orthogonalen $U_{i j}$ Tensors.

Halogen-Metall-Austausch zu $\left(\mathrm{C}_{5} \mathrm{Cl}_{4} \mathrm{Li}\right) \mathrm{Rh}(\mathrm{COD})(\mathbf{4 b})$ und anschließend mit diversen Elektrophilen in meist guter Ausbeute die Verbindungen 4c-4g.

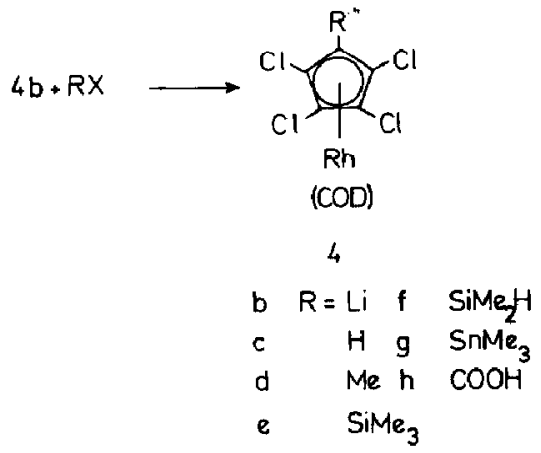

Von der Reaktion mit Disulfiden haben wir bereits an anderer Stelle berichtet [1]. Die Reaktion mit $\mathrm{CO}_{2}$ und anschließender Protonierung ergibt eine sehr instabile Verbindung, vermutlich die Carboxyverbindung $4 \mathrm{~b}$. Diese lagert sich anscheinend in eine Verbindung um, die wohl nicht mehr einen $\pi$-gebundenen Fünfring, sondern eine Koordination über eine doppelt verbrückende Carboxylgruppe aufweist.

\section{Experimenteller Teil}

Die Ausgangsverbindungen 1a, 3a, 4a, 4b wurden wie früher beschrieben dargestellt. Alle Reaktionen wurden in Schlenkrohren in $\mathrm{N}_{2}$-Atmosphäre durchgeführt, die Lösungsmittel nach den üblichen Verfahren absolutiert und die verwendeten Reagenzien waren handelsübliche Produkte. NMR-Spektren wurden an einem 
Tabelle 2

Wichtige Bindungslängen $(\mathrm{pm})$ und Winkel $\left({ }^{\circ}\right)$ von $3 \mathrm{e}$

\begin{tabular}{llll}
\hline$M n(1)-C(11)$ & $212.9(8)$ & $M n(1)-C(12)$ & $215.3(10)$ \\
$M n(1)-C(13)$ & $213.9(9)$ & $M n(1)-C(14)$ & $213.3(7)$ \\
$M n(1)-C(15)$ & $213.6(8)$ & $C(11)-C(12)$ & $143.7(12)$ \\
$C(11)-C(15)$ & $142.3(12)$ & $C(11)-C(16)$ & $151.1(10)$ \\
$C(12)-C(13)$ & $139.4(11)$ & $C(12)-C l(12)$ & $172.2(10)$ \\
$C(13)-C(14)$ & $140.3(13)$ & $C(13)-C l(13)$ & $171.9(8)$ \\
$C(14)-C(15)$ & $141.9(10)$ & $C(14)-C l(14)$ & $171.7(8)$ \\
$C(15)-C l(15)$ & $170.8(9)$ & $C(16)-O(4)$ & $123.9(10)$ \\
$C(16)-N(1)$ & $130.4(14)$ & & \\
$C(12)-C(11)-C(15)$ & $106.2(6)$ & $C(12)-C(11)-C(16)$ & $127.5(8)$ \\
$C(15)-C(11)-C(16)$ & $126.2(8)$ & $C(11)-C(12)-C(13)$ & $108.4(8)$ \\
$C(11)-C(12)-C l(12)$ & $125.6(6)$ & $C(13)-C(12)-C l(12)$ & $125.9(7)$ \\
$C(12)-C(13)-C(14)$ & $109.1(7)$ & $C(12)-C(13)-C l(13)$ & $125.6(8)$ \\
$C(14)-C(13)-C l(13)$ & $124.9(6)$ & $C(13)-C(14)-C(15)$ & $107.6(7)$ \\
$C(13)-C(14)-C l(14)$ & $126.4(6)$ & $C(15)-C(14)-C l(14)$ & $125.5(7)$ \\
$C(11)-C(15)-C(14)$ & $108.6(8)$ & $C(11)-C(15)-C l(15)$ & $125.4(5)$ \\
$C(14)-C(15)-C l(15)$ & $125.8(7)$ & $C(11)-C(16)-O(4)$ & $120.1(8)$ \\
$C(11)-C(16)-N(1)$ & $116.5(7)$ & $O(4)-C(16)-N(1)$ & $123.4(7)$ \\
\hline
\end{tabular}

JEOL-FX 90-NMR-Gerät aufgenommen. Chemische Verschiebungen wurden auf die Signale der Lösungsmittel bezogen $\left(\mathrm{C}_{6} \mathrm{D}_{6}:{ }^{1} \mathrm{H}: \delta=7.15 \mathrm{ppm} ;{ }^{13} \mathrm{C}: \delta=128.0\right.$ ppm). IR-Spektren in Hexan, NMR-Spektren in $\mathrm{C}_{6} \mathrm{D}_{6}$, wenn nicht anders an gegeben.

Tricarbonyl(tetrachlorotrimethylstannylcyclopentadienyl)mangan (2a)

$300 \mathrm{mg} 1 \mathrm{a}(0.71 \mathrm{mmol})$ werden in $10 \mathrm{ml} \mathrm{Et}{ }_{2} \mathrm{O}$ bei $-60^{\circ} \mathrm{C}$ mit $0.44 \mathrm{ml} 1.6 \mathrm{~m}$ BuLi-Lösung $15 \mathrm{~min}$ gerührt und dann mit $141 \mathrm{mg} \mathrm{SnMe} \mathrm{Cl}_{3}(0.71 \mathrm{mmol})$ versetzt. Es wird langsam auf RT erwärmt und das Lösungsmittel i.Vak. entfernt. Der Rückstand wird mit zweimal je $10 \mathrm{ml}$ Hexan extrahiert und über eine kurze Kieselgelsäule $(2 \times 2 \mathrm{~cm})$ filtriert. Es wird mit Hexan nachgewaschen, die geammelten Eluate werden zur Trockene gebracht. Es bleibt ein gelbes Pulver zurück. Ausb.: $200 \mathrm{mg}$ (56\%). Schmp. $68-69^{\circ} \mathrm{C}$. IR: $2038 \mathrm{~cm}^{-1}, 1968(\nu(\mathrm{CO})) ;{ }^{1} \mathrm{H}-\mathrm{NMR}: \delta=0.29$ ppm $\left(\mathrm{SnCH}_{3}\right) ;{ }^{13} \mathrm{C}-\mathrm{NMR}: \delta=105.4,98.2,76.2 \mathrm{ppm}\left(\mathrm{C}_{5} \mathrm{R}_{5}\right),-6.71\left(\mathrm{SnCH}_{3}\right)$. Gef.: C, 26.48; $\mathrm{H}, 2.12 . \mathrm{C}_{11} \mathrm{H}_{9} \mathrm{Cl}_{4} \mathrm{MnO}_{3} \mathrm{Sn}$ (504.63) ber.: C, 26.18; $\mathrm{H}, 1.80 \%$.

\section{Tricarbonyl(tetrachlordiphenylphosphinocyclopentadienyl)mangan (2b)}

Aus $435 \mathrm{mg} 1 \mathrm{a}(1.04 \mathrm{mmol})$ und $0.64 \mathrm{ml}$ BuLi-Lösung (1.04 mmol) wird wie unter 2a beschrieben eine Lösung von $1 b$ erstellt, die mit $0.19 \mathrm{ml} \mathrm{PPh}_{2} \mathrm{Cl}$ versetzt und auf RT gebracht wird. Es wird zentrifugiert und der farblose Niederschlag abgetrennt. Nach Abziehen des Lösungsmittel wird mit $15 \mathrm{ml}$ Pentan gewaschen, zentrifugiert und mit $5 \mathrm{ml} \mathrm{CH}_{2} \mathrm{Cl}_{2}$ extrahiert. Der Extrakt wird mit $20 \mathrm{ml}$ Pentan versetzt und über Nacht auf $-20^{\circ} \mathrm{C}$ gekühlt. Der dabei entstehende beige Niederschlag wird isoliert und bei RT mit wenig Hexan gewaschen, worauf ein farbloses Pulver zurückbleibt. Ausb. $197 \mathrm{mg}$ (36\%). Schmp. 141-143 ${ }^{\circ} \mathrm{C}$. IR: $2051 \mathrm{~cm}^{-1}, 1992,1974$ $\left(\nu(\mathrm{CO})\right.$ ); ${ }^{13} \mathrm{C}$-NMR (Aceton- $\left.d_{6}\right): \delta=104.0,98.0,86.5 \mathrm{ppm}\left(\mathrm{C}_{5} \mathrm{R}_{5}\right)$. Gef.: C, 45.57; $\mathrm{H}, 2.26 . \mathrm{C}_{20} \mathrm{H}_{10} \mathrm{Cl}_{4} \mathrm{MnO}_{3} \mathrm{P}$ (526.02) ber.: C, 45.67; H, 1.92\%. 


\section{Tricarbonyl(tetrachlorphenylselenocyclopentadienyl)mangan (2c)}

$655 \mathrm{mg} 1 \mathrm{a}(1.56 \mathrm{mmol})$ werden in $10 \mathrm{ml} \mathrm{Et}{ }_{2} \mathrm{O}$ bei $-40^{\circ} \mathrm{C}$ mit $1.0 \mathrm{ml} \mathrm{BuLi}$-Lösung (1.6 mmol) $20 \mathrm{~min}$ gerührt und dann mit $450 \mathrm{mg} \mathrm{Ph}_{2} \mathrm{Se}_{2}(1.4 \mathrm{mmol})$ versetzt. Nach weiteren $15 \mathrm{~min}$ wird auf RT gebracht und nach $90 \mathrm{~min}$ das Lösungsmittel abgezogen. Das zurückbleibende rotbraune Öl wird mit zweimal $20 \mathrm{ml}$ Hexan extrahiert; die Extrakte werden über Kieselgel $(2 \times 4 \mathrm{~cm})$ filtriert. Es wird mit weiteren $20 \mathrm{ml}$ Hexan eluiert, die vereinigten Eluate werden auf $10 \mathrm{ml}$ eingeengt und dann auf $-78^{\circ} \mathrm{C}$ gekühlt. Das ausgefallene Produkt wird isolicrt und getrocknet. Ausb. $465 \mathrm{mg}(60 \%)$. IR: $2046 \mathrm{~cm}^{-1}, 1980(\nu(\mathrm{CO})) ;{ }^{13} \mathrm{C}$-NMR: $\delta=105.7,96.1,77.9$ ppm $\left(\mathrm{C}_{5} \mathrm{R}_{5}\right)$. Gef.: $\mathrm{C}, 34.98 ; \mathrm{H}, 1.40 . \mathrm{C}_{14} \mathrm{H}_{5} \mathrm{Cl}_{4} \mathrm{MnO}_{3} \mathrm{Se}(496.89)$ ber.: $\mathrm{C}, 33.8 ; \mathrm{H}$, $1.01 \%$.

\section{Bis(tricarbonyltetrachlorcyclopentadienylmangan)sulfid (2d)}

Wie üblich wird aus $840 \mathrm{mg} \mathrm{1a}(2.0 \mathrm{mmol})$ und $1.25 \mathrm{ml}$ BuLi-Lösung bei $-60^{\circ} \mathrm{C}$ in $10 \mathrm{ml} \mathrm{Et}{ }_{2} \mathrm{O}$ eine Lösung von $1 \mathrm{~b}$ hergestellt. Nach 10 min wird mit $0.06 \mathrm{ml}^{\mathrm{SCl}} \mathrm{Sl}_{2}$ $(1.0 \mathrm{mmol})$ versetzt und über Nacht auf RT gebracht. Es wird wie immer aufgearbeitet und zum Schluß aus Hexan umkristallisiert. Ausb. $100 \mathrm{mg}$ (14\%). Schmp. $178-180^{\circ} \mathrm{C}$ (Zers.). IR: 2042vs, 2038m, 1987vs, $1952 \mathrm{w} \mathrm{cm}{ }^{-1}$ ( $\nu(\mathrm{CO})$ ). ${ }^{13} \mathrm{C}-\mathrm{NMR}$ : $\delta=103.9$, 96.5, 83.1 ppm $\left(\mathrm{C}_{5} \mathrm{R}_{5}\right)$. Gef.: $\mathrm{C}, 26.51 ; \mathrm{H}, 0.21 ; \mathrm{S}, 5.22 . \mathrm{C}_{16} \mathrm{Cl}_{8} \mathrm{Mn}_{2} \mathrm{O}_{6} \mathrm{~S}$ (713.74) ber.: C, 26.93; H, 0.00; S, 4.49\%.

\section{Tricarbonyl(tetrachloroformylcyclopentadienyl)mangan (2e)}

$583 \mathrm{mg} \mathrm{1a}, 0.87 \mathrm{ml}$ BuLi-Lösung (jeweils $1.39 \mathrm{mmol}$ ) und $0.1 \mathrm{ml}$ Dimethylformamid werden in $10 \mathrm{ml} \mathrm{Et}{ }_{2} \mathrm{O}$ bei $-60^{\circ} \mathrm{C}$ zur Reaktion gebracht. Innerhalb $4 \mathrm{~h}$ wird auf RT gebracht und dann das Lösungsmittel im Vak. entfernt. Das zurückbleibende braune Öl wird nacheinander mit $15 \mathrm{ml} 2 n-\mathrm{HCl}$ und $20 \mathrm{ml}$ Toluol versetzt. Die orangebraune Toluolphase wird abgetrennt und zur Trockene gebracht. Es wird mit $10 \mathrm{ml}$ Hexan ausgeruhrt, vom Ungelösten abgetrennt, auf $3 \mathrm{ml}$ eingeengt und an Silicagel $(5 \times 2 \mathrm{~cm})$ chromatographiert. Das Eluat mit $\mathrm{Et}_{2} \mathrm{O}$ wird zur Trockene gebracht, in wenig Hexan aufgenommen und durch Abkühlen auf $-30^{\circ} \mathrm{C}$ zur Kristallisation gebracht. Das gelbe Pulver wird isoliert und getrocknet. Ausb. $235 \mathrm{mg}$ (46\%). Schmp. 64-65 ${ }^{\circ}$ C. IR: $2051 \mathrm{~cm}^{-1}, 1992,1984$ ( $\nu$ (MCO)), 1708 $(\nu(\mathrm{CHO})) .{ }^{13} \mathrm{C}-\mathrm{NMR}: \delta=182.6(\mathrm{CHO}), 100.4,98.0,76.8\left(\mathrm{C}_{5} \mathrm{R}_{5}\right)$.

\section{Umsetzung von $1 \mathrm{~b}$ mit $N, N$-Bis(trimethylsilyl)-O-mesitylensulfonyl-hydroxylamin}

$900 \mathrm{mg} 1 \mathrm{a}(2.14 \mathrm{mmol})$ werden in $15 \mathrm{ml} \mathrm{Et}{ }_{2} \mathrm{O}$ bei $-60^{\circ} \mathrm{C}$ mit zuerst $1.34 \mathrm{ml}$ BuLi-Lösung (2.14 mmol) und dann $770 \mathrm{mg} \mathrm{MesSO} \mathrm{ON}_{2} \mathrm{ON}\left(\mathrm{SiMe}_{3}\right)_{2}(2.14 \mathrm{mmol})$ versetzt. Nachdem auf RT erwärmt wird, wird das Lösungsmittel i.Vak. abgezogen, und der Rückstand wird zweimal mit je $20 \mathrm{ml}$ Hexan verrührt. Der hexanlösliche Teil wird mit $20 \mathrm{ml}$ Wasser gewaschen, mit $\mathrm{MgSO}_{4}$ getrocknet, filtriert und zur Trockene gebracht. Es wird mit je $10 \mathrm{ml} \mathrm{Et}_{2} \mathrm{O}$ und $\mathrm{H}_{2} \mathrm{O}$ sowie einigen Tropfen konz. $\mathrm{HCl}$ versetzt, wobei sich die Etherphase orange färbt. Diese wird abgetrennt, mit $\mathrm{MgSO}_{4}$ getrocknet, filtriert und zur Trockene eingedampft. Nach erneuter Zugabe von Hexan wird ein farbloser Niederschlag erhalten (laut IR-Spektrum handelt es sich um $\mathrm{MesSO}_{2} \mathrm{ONH}_{2}$ ) und eine orange Lösung, die nach Abziehen des Lösungsmittels im Vakuum lediglich $\left(\mathrm{C}_{5} \mathrm{Cl}_{4} \mathrm{H}\right) \mathrm{Mn}(\mathrm{CO})_{3}$ liefert. 
(Aminotetrachloracyclopentadienyl)tricarbonylmangan (2f)

Ca. $500 \mathrm{mg} \mathrm{3c}$ ( $\leq 1.22 \mathrm{mmol}$, sh. unten) werden in $20 \mathrm{ml}$ Methylcyclohexan 30 min bei $80^{\circ} \mathrm{C}$ gerührt. Es wird über $H y$ flo-kieselgur filtriert und das Filtrat zur Trockene gebracht. Das zurückbleibende Öl wird mit zweimal je $10 \mathrm{ml}$ Hexan ausgerührt. Es wird vom Ungelösten abgetrennt und zur Trockene gebracht. Der Rückstand (3d) wird in $20 \mathrm{ml} 10 \%$ iger wäßriger Kalilauge $30 \mathrm{~min}$ am Rückfluss gekocht und nach dem Erkalten mit $30 \mathrm{ml} \mathrm{Et}{ }_{2} \mathrm{O}$ extrahiert. Die abgetrennte Etherphase wird mit $\mathrm{MgSO}_{4}$ getrocknet, filtriert und zur Trockene gebracht. Ausb. $50 \mathrm{mg}(\approx 11 \%)$. IR $\left(\mathrm{CH}_{2} \mathrm{Cl}_{2}\right): 2043 \mathrm{sh}, 2033 \mathrm{~cm}^{-1}, 1958$ ( $(\mathrm{CO})$ ); (Nujol): 3490m, 3392s $\left(\nu\left(\mathrm{NH}_{2}\right)\right) .{ }^{13} \mathrm{C}-\mathrm{NMR}: \delta=118.3,94.2,76.8 \mathrm{ppm}\left(\mathrm{C}_{5} \mathrm{R}_{5}\right)$.

\section{Tricarbonyl(tetrachlorocyanocyclopentadienyl)mangan (2g)}

$250 \mathrm{mg} 3 \mathrm{e}\left(0.6 \mathrm{mmol}\right.$, sh. unten) werden in ca. $5 \mathrm{ml} \mathrm{POCl}{ }_{3}$ gelöst und bei $120^{\circ} \mathrm{C}$ 15 min gerührt. Nach dem Abkühlen auf RT wird vorsichtig in $4^{\circ} \mathrm{C}$ kaltes Wasser eingetropft und dreimal mit je $10 \mathrm{ml} \mathrm{Et}{ }_{2} \mathrm{O}$ extrahiert. Die vereinigten Etherphasen werden über $\mathrm{MgSO}_{4}$ getrocknet. Nach Filtrieren und Abziehen des Lösungsmittels i.Vak. hinterbleibt ein gelbes Pulver. Schmp. 73-75 ${ }^{\circ} \mathrm{C}$ (Zers.). IR: $2055 \mathrm{~cm}^{-1}, 1995$, $1991(\nu(\mathrm{CO}))$, 2246vw ( $\nu(\mathrm{CN}))$. ${ }^{13} \mathrm{C}-\mathrm{NMR}: \delta=100.5,96.6,61.0 \mathrm{ppm}\left(\mathrm{C}_{5} \mathrm{R}_{5}\right)$. Gef.: $\mathrm{C}, 29.89 ; \mathrm{H}, 0.68 ; \mathrm{N}, 3.69 . \mathrm{C}_{9} \mathrm{Cl}_{4} \mathrm{MnNO}_{3}$ (366.86) ber.: C, 29.47; H, 0.00; N, 3.82\%.

\section{Tricarbonyl(chlorocarbonyltetrachlorocyclopentadienyl)mangan (3b)}

$360 \mathrm{mg} 3 \mathrm{a}(0.94 \mathrm{mmol})$ werden in $20 \mathrm{ml} \mathrm{CH} \mathrm{Cl}_{2}$ mit $0.08 \mathrm{ml}$ Oxalylchlorid (0.94 mmol) und I Tropfen Dimethylformamid $60 \mathrm{~h}$ bei RT gerürt. Nach dem Abziehen des Lösungsmittels i.Vak. wird der Rückstand mit $20 \mathrm{ml}$ Hexan extrahiert. Der zur Trockene eingeengte Extrakt liefert ein gelbes Pulver. Ausb. $345 \mathrm{mg}$ (91\%). Schmp. $60^{\circ} \mathrm{C}$ (Zers.). IR: $2056 \mathrm{~cm}^{-1}, 1997,1992$ ( $\nu(\mathrm{MCO})$ ), 1719, 1673 ( $\left.\nu(\mathrm{COCl})\right) .{ }^{13} \mathrm{C}-$ NMR (Aceton- $d_{6}$ ): $\delta=160.7(\mathrm{COCl}), 101.7,98.6,77.4 \mathrm{ppm}\left(\mathrm{C}_{5} \mathrm{R}_{5}\right)$. Gef.: C, 27.32; $\mathrm{H}, 0.15$. $\mathrm{C}_{9} \mathrm{Cl}_{5} \mathrm{MnO}_{4}$ (404.30) ber.: C, 26.74; $\mathrm{H}, 0.00 \%$.

\section{(Azidocarbonyltetrachlorocyclopentadienyl)tricarbonylmangan (3c)}

$800 \mathrm{mg} 3 \mathrm{~b}(1.98 \mathrm{mmol})$ werden in $20 \mathrm{ml}$ Aceton gelöst, auf $-20^{\circ} \mathrm{C}$ gekühlt und portionsweise mit $100 \mathrm{mg} \mathrm{NaN}$ (1.54 mmol) versetzt. Nach $45 \mathrm{~min}$ Rühren bei $0^{\circ} \mathrm{C}$ werden $5 \mathrm{ml} \mathrm{H}{ }_{2} \mathrm{O}$ zugegeben. Man rührt dann weitere $15 \mathrm{~min}$. Anschließend wird mit zweimal je $10 \mathrm{ml} \mathrm{Et}{ }_{2} \mathrm{O}$ extrahiert. Die Etherextrakte werden uber $\mathrm{Na}_{2} \mathrm{SO}_{4}$ getrocknet, filtriert und vollständig i.Vak. eingedampft. Der Rückstand wird mit 20 ml Hexan verrührt, filtriert und zur Trockene gebracht. Ausb. $520 \mathrm{mg}$ (64\%). Schmp. 51-53 ${ }^{\circ} \mathrm{C}$ (Zers.). IR: $2053 \mathrm{~cm}^{-1}, 1991,1986$ ( $\left.\nu(\mathrm{MCO})\right), 2260\left(\nu\left(\mathrm{N}_{3}\right)\right), 1703$ $\left(\nu\left(\mathrm{CON}_{3}\right)\right.$ ). Gef.: $\mathrm{C}, 26.04 ; \mathrm{H}, 0.56 ; \mathrm{N}, 10.10 . \mathrm{C}_{9} \mathrm{Cl}_{4} \mathrm{MnN}_{3} \mathrm{O}_{4}$ (410.87) ber.: $\mathrm{C}$, $26.31 ; \mathrm{H}, 0.00 ; \mathrm{N}, 10.23 \%$.

\section{(Amidotetrachlorocyclopentadienyl)tricarbonylmangan (3e)}

$150 \mathrm{mg} 3 b(0.37 \mathrm{mmol})$ werden in $15 \mathrm{ml}$ Hexan gelöst und $1 \mathrm{~h}$ in einer $\mathrm{N}_{2} / \mathrm{NH}_{3}$-Atmosphäre gerührt. Der ausgefallene farblose Niederschlag wird durch Zentrifugieren isoliert, mit Wasser gewaschen und über $\mathrm{P}_{2} \mathrm{O}_{5}$ getrocknet. Ausbeute $130 \mathrm{mg}$ (91\%). Schmp. $131-133^{\circ} \mathrm{C}$. Form I: IR $\left(\mathrm{CH}_{2} \mathrm{Cl}_{2}\right): 2046 \mathrm{~cm}^{-1}, 1978$ $(\nu(\mathrm{MCO})) ;(\mathrm{Nujol}): 1694,1586\left(\nu\left(\mathrm{CONH}_{2}\right)\right) .{ }^{13} \mathrm{C}-\mathrm{NMR}:\left(\right.$ Aceton- $\left.d_{6}\right): \delta=159.7$ $\left(\mathrm{CONH}_{2}\right)$, 97.4, 95.8, 88.6 ppm $\left(\mathrm{C}_{5} \mathrm{R}_{5}\right)$. Gef.: C, 28.09; $\mathrm{H}, 0.71 ; \mathrm{N}, 3.72$. $\mathrm{C}_{9} \mathrm{H}_{2} \mathrm{Cl}_{4} \mathrm{MnNO}_{4}$ (384.87) ber.: C, 28.09; $\mathrm{H}, 0.52 ; \mathrm{N}, 3.64 \%$. 


\section{Umsetzung von 3e mit Tosylchlorid und Pyridin}

$430 \mathrm{mg} 3 \mathrm{e}(1.12 \mathrm{mmol})$ werden bei $0^{\circ} \mathrm{C}$ in $5 \mathrm{ml}$ Pyridin mit einer Lösung von 230 mg Tosylchlorid $(1.20 \mathrm{mmol})$ in $2 \mathrm{ml}$ Pyridin versetzt. Nach 15 min wird in $20 \mathrm{ml}$ Wasser eingegossen, kurz gerührt und dann mit $20 \mathrm{ml} \mathrm{Et}{ }_{2} \mathrm{O}$ überschichtet. Die nun gelbe etherische Phase wird abgetrennt, mit $\mathrm{MgSO}_{4}$ getrocknet und anschließend zur Trockene eingedampft. Es wird mit $20 \mathrm{ml}$ Hexan versetzt, wobei eine gelbe Lösung und ein gelber Niederschlag erhalten werden. Der Niederschlag wird isoliert und getrocknet. Ausb. $305 \mathrm{mg}$. Schmp. $133-135^{\circ} \mathrm{C}$. Aus der gelben Hexanlösung werden Kristalle erhalten, die für die Röntgenstrukturanalyse verwendet werden. Form II: IR (Nujol): $1708 \mathrm{w}, 1655 \mathrm{vs}, 1608 \mathrm{~m} \mathrm{~cm}^{-1}\left(\nu\left(\mathrm{CONH}_{2}\right)\right)$.

\section{Umsetzung von la mit CuCN in Pyridin}

$112 \mathrm{mg} 1 \mathrm{a}$ werden mit $1 \mathrm{~g} \mathrm{CuCN}$ in $20 \mathrm{ml}$ Pyridin $90 \mathrm{~min}$ am Rückfluss gekocht, wobei sich die Lösung schwarz färbt. Nach dem Abkühlen wird $20 \mathrm{ml}$ Hexan zugesetzt, kurz gerührt und abgefrittet. Der Rückstand auf der Fritte wird mit $20 \mathrm{ml}$ Pyridin extrahiert. Durch eindiffundierendes Pentan wird im Extrakt ein erster schwarzer Niederschlag erhalten, der abgetrennt wird. In der filtrierten Fällungslösung läßt sich mit weiterem Pentan ein grüner Niederschlag fällen, aus dem nach erneuter Filtration mit heißem Acetonitril $\mathrm{Cu}$ (py) ${ }_{2} \mathrm{Br}_{2}$ und $\mathrm{Cu}(\mathrm{py})_{2} \mathrm{Cl}_{2}$ (durch IR-Spektren und $\mathrm{C}-\mathrm{H}-\mathrm{N}$-Analyse charakterisiert) extrahiert werden können. Erneutes Auflösen des Extraktionsrückstandes in Pyridin liefert nach Eindiffusion von Pentan einige wenige blaue Kristalle. Eine Kristallstrukturanalyse erweist diese als polymeres $\left[(\mathrm{NC}) \mathrm{Cu}(\mathrm{py})_{2}(\mathrm{CN}) \mathrm{Cu}(\mathrm{py})_{2} \mathrm{Cl}\right]_{x}$.

\section{(Tetrachlorcyclopentadienyl)(cyclooctadien)rhodium (4c)}

$300 \mathrm{mg} 4 \mathrm{a}(0.67 \mathrm{mmol})$ werden in $10 \mathrm{ml} \mathrm{Et}_{2} \mathrm{O}$ bei $-60^{\circ} \mathrm{C}$ mit $0.42 \mathrm{ml}$ BuLi-Lösung versetzt. Nach einigen Minuten werden einige Tropfen $\mathrm{H}_{2} \mathrm{O}$ zugegeben und innerhalb $2 \mathrm{~h}$ auf RT gebracht. Das Lösungsmittel wird abgezogen und der Rückstand mit $20 \mathrm{ml}$ Pentan ausgewaschen. Die Pentanlösung wird zur Trockene gebracht. ${ }^{1} \mathrm{H}-\mathrm{NMR}: \delta=5.29 \mathrm{~d}\left({ }^{2} J\left({ }^{103} \mathrm{Rh}-{ }^{1} \mathrm{H}\right)=0.8 \mathrm{~Hz}, \mathrm{C}_{5} \mathrm{H}\right), 3.66 \mathrm{~s}(=\mathrm{CH}), 1.92 \mathrm{~m}$ $\left(-\mathrm{CH}_{2}\right) .{ }^{13} \mathrm{C}-\mathrm{NMR}$ (hier und im folgenden nur Resonanzen des Cyclopentadienylrings): $\delta=96.5 \mathrm{~d}\left({ }^{1} J\left({ }^{103} \mathrm{Rh}-{ }^{13} \mathrm{C}\right)=3.7 \mathrm{~Hz}\right), 93.8 \mathrm{~d}(4.3 \mathrm{~Hz}), 85.4 \mathrm{~d}(3.6 \mathrm{~Hz})$.

\section{(Tetrachlormethylcyclopentadienyl)(cyclooctadien)rhodium (4d)}

$335 \mathrm{mg} 4 \mathbf{a}(0.75 \mathrm{mmol})$ werden bei $-60^{\circ} \mathrm{C}$ in $10 \mathrm{ml} \mathrm{Et}_{2} \mathrm{O}$ mit $0.47 \mathrm{ml}$ BuLi-Lösung $(0.75 \mathrm{mmol})$ und $0.05 \mathrm{ml} \mathrm{MeI}(0.75 \mathrm{mmol})$ zur Reaktion gebracht. Nachdem die Temperatur auf RT angestiegen ist, wird das Lösungsmittel abgezogen und der Rückstand mit zweimal $10 \mathrm{ml}$ Hexan extrahiert. Die vereinigten Extrakte werden bis auf $2 \mathrm{ml}$ eingeengt und an einer Kieselgelsäule $(5 \times 2 \mathrm{~cm})$ chromatographiert. Das Eluat mit Hexan wird zur Trockene gebracht. Ausb.: $70 \mathrm{mg}$ (22\%). Schmp. $70^{\circ} \mathrm{C}$ (Zers.). Gef.: C, 37.82; H, 3.28. $\mathrm{C}_{14} \mathrm{H}_{15} \mathrm{Cl}_{4} \mathrm{Rh}$ (428.00) ber.: C, $39.29 ; \mathrm{H}, 3.53 \%$.

\section{(Tetrachlortrimethylsilylcyclopentadienyl)(cyclooctadien)rhodium (4e)}

$530 \mathrm{mg} 4 \mathrm{a}(1.18 \mathrm{mmol})$ werden in $10 \mathrm{ml} \mathrm{Et}{ }_{2} \mathrm{O}$ bei $-60^{\circ} \mathrm{C}$ mit $0.74 \mathrm{ml}$ BuLi-Lösung (1.18 mmol) $30 \mathrm{~min}$ gerührt. Nach Zugabe von $0.16 \mathrm{ml} \mathrm{SiMe} \mathrm{Cl}_{3} \mathrm{Cl}(1.18$ mmol) wird die Lösung langsam auf $-20^{\circ} \mathrm{C}$ erwärmt und dann bei dieser Temperatur das Lösungsmittel i. Vak. entfernt. Weitere Aufarbeitung wie bei 4d. Ausb.: 490 
mg (85\%). Schmp. $104-06^{\circ} \mathrm{C} .{ }^{1} \mathrm{H}-\mathrm{NMR}: \delta=0.42 \mathrm{~s}\left(\mathrm{SiCH}_{3}\right), 3.62 \mathrm{~s}(=\mathrm{CH}), 1.98 \mathrm{~m}$ $\left(\mathrm{CH}_{2}\right) .{ }^{13} \mathrm{C}-\mathrm{NMR}: \delta=99.6 \mathrm{~d}(3.6 \mathrm{~Hz}), 98.9 \mathrm{~d}(4.4 \mathrm{~Hz}), 92.0 \mathrm{~d}(2.6 \mathrm{~Hz})$. Gef.: $\mathrm{C}$, 39.03; H, 4.37. $\mathrm{C}_{16} \mathrm{H}_{21} \mathrm{Cl}_{4} \mathrm{RhSi}(486.15)$ ber.: C, 39.53; H, 4.35\%.

\section{(Tetrachlordimethylsilylcyclopentadienyl)(cyclooctadien)rhodium (\$f)}

$500 \mathrm{mg} 4 \mathrm{a}(1.11 \mathrm{mmol})$ werden in $10 \mathrm{ml} \mathrm{Et}{ }_{2} \mathrm{O}$ bei $-60^{\circ} \mathrm{C}$ mit $0.69 \mathrm{ml}$ BuLi-Lösung (1.11 mmol) $15 \mathrm{~min}$ gerührt. Nach Zugabe von $0.12 \mathrm{ml} \mathrm{SiMe} \mathrm{SCl}_{2} \mathrm{HCl}$ (1.11 mmol) wird während $2.5 \mathrm{~h}$ auf $-30^{\circ} \mathrm{C}$ erwärmt und dann bei dieser Temperatur das Lösungsmittel i.Vak. entfernt. Der Rückstand wird wie üblich aufgearbeitet. Ausb.: $460 \mathrm{mg}$ (87\%). Schmp. $70-71^{\circ} \mathrm{C}$. IR (Nujol): $2145 \mathrm{~cm}^{-1}$ $(\nu(\mathrm{SiH})) .{ }^{1} \mathrm{H}-\mathrm{NMR}: \delta=4.78 \mathrm{sp}\left({ }^{3} J\left({ }^{1} \mathrm{H}-{ }^{1} \mathrm{H}\right)=3.8 \mathrm{~Hz}\right)(\mathrm{SiH}), 0.42 \mathrm{~d}(3.9 \mathrm{~Hz})\left(\mathrm{SiCH}_{3}\right)$, $3.62 \mathrm{~s}(=\mathrm{CH}), 1.95 \mathrm{~m}\left(\mathrm{CH}_{2}\right) \cdot{ }^{13} \mathrm{C}-\mathrm{NMR}: \delta=99.5 \mathrm{~d}(3.0 \mathrm{~Hz}), 99.0 \mathrm{~d}(4.4 \mathrm{~Hz}), 89.9 \mathrm{~d}$ $(2.9 \mathrm{~Hz})\left(\mathrm{C}_{5} \mathrm{R}_{5}\right)$. Gef.: C, 37.99; $\mathrm{H}, 4.03 . \mathrm{C}_{15} \mathrm{H}_{19} \mathrm{Cl}_{4} \mathrm{RhSi}(472.12)$ ber.: C, 38.16; $\mathrm{H}$, 4.06\%.

(Tetrachlorotrimethylstannylcyclopentadienyl)(cyclooctadien)rhodium (4g)

$570 \mathrm{mg} 4 \mathrm{a}(1.27 \mathrm{mmol})$ werden in $15 \mathrm{ml} \mathrm{Et}{ }_{2} \mathrm{O}$ bei $-60^{\circ} \mathrm{C}$ mit $0.8 \mathrm{ml} 1.6 \mathrm{~m}$ BuLi-Lösung $(1.27 \mathrm{mmol})$ versetzt und $5 \mathrm{~min}$ gerührt. Dann wird $0.25 \mathrm{~g} \mathrm{SnMe} \mathrm{Sl}_{3}$ (1.27 mmol) zugegeben und innerhalb $3 \mathrm{~h}$ auf $-10^{\circ} \mathrm{C}$ gebracht. Bei dieser Temperatur wird das Lösungsmittel i.Vak. abgezogen. Der Rückstand wird bei RT zweimal mit je $15 \mathrm{ml}$ Hexan extrahiert und wie üblich aufgearbeitet. Ausb.: $330 \mathrm{mg}$ (45\%). Schmp. $102-104^{\circ} \mathrm{C}$ (Zers.). ${ }^{1} \mathrm{H}-\mathrm{NMR}: \delta=0.41 \mathrm{~s}\left(\mathrm{SnCH}_{3}\right), 3.65 \mathrm{~s} \quad(=\mathrm{CH}), 1.99 \mathrm{~m}$ $\left(\mathrm{CH}_{2}\right),{ }^{13} \mathrm{C}-\mathrm{NMR}: \delta=100.0 \mathrm{~d}(5.1 \mathrm{~Hz}), 98.9 \mathrm{~d}(3.6 \mathrm{~Hz}), 90.6 \mathrm{~d}(3.0 \mathrm{~Hz})$. Gef.: C, 33.27; H, 3.59. $\mathrm{C}_{16} \mathrm{H}_{21} \mathrm{Cl}_{4} \mathrm{RhSn}$ (576.66) ber.: C, 33.33; $\mathrm{H}, 3.67 \%$.

\section{(Carboxytetrachlorcyclopentadienyl)(cyclooctadien)rhodium (4h)}

$640 \mathrm{mg} 4 \mathrm{a}(1.43 \mathrm{mmol})$ werden in $15 \mathrm{ml} \mathrm{Et}_{2} \mathrm{O}$ bei $-60^{\circ} \mathrm{C}$ mit $0.89 \mathrm{ml}$ BuLi-Lösung (1.43 mmol) $15 \mathrm{~min}$ gerührt. Dann wird ein Stückchen Trockeneis zugesetzt und langsam auf RT etwärmt. Der entstandene Niederschlag wird isoliert und mit einer Mischung von $10 \mathrm{ml} \mathrm{Et}{ }_{2} \mathrm{O}$ und $20 \mathrm{ml} \mathrm{H}{ }_{2} \mathrm{O}$ behandelt. Die abgetrennte wäßrige Phase wird mit $20 \mathrm{ml} \mathrm{Et}{ }_{2} \mathrm{O}$ und $2 \mathrm{ml}$ konz. $\mathrm{HCl}$ gerührt. Die nun orange $\mathrm{Et}_{2} \mathrm{O}$-Phase wird über $\mathrm{MgSO}_{4}$ getrocknet, filtriert und i.Vak. eingedampft. Das zurückbleibende braune Öl wird durch Verrühren mit Hexan zur Kristallisation gebracht. Weitere Reinigungsversuche durch Umkristallisation aus polaren Lösungsmitteln führten zur Schwarzfärbung und Zersetzung des Produktes. Im ${ }^{1} \mathrm{H}$-NMR-Spektrum verliert das $=\mathrm{CH}$-Signal bei $\delta=3.68 \mathrm{ppm}$ an Intensität und ein neues Signal bei 4.29 ppm entsteht. Während das erste Signal ähnlich denen der anderen Verbindungen 4 ist, entspricht das zweite Verbindungen vom Typ $[(\mathrm{COD}) \mathrm{RhCl}]_{2}$.

\section{Dank}

Herrn Prof. Dr. W. Beck danken wir für sein förderndes Interesse und die Unterstützung mit Institutsmitteln.

\section{Literatur}

1 IV. Mitteilung: K. Sünkel und D. Steiner, Chem. Ber., im Druck.

2 D.W. Macomber, W.P. Hart und M.D. Rausch, Adv. Organomet. Chem., 21 (1982) 1. 
3 (a) K. Sünkel und D. Motz, Chem. Ber., 121 (1988) 799; (b) K. Sünkel und D. Motz, Angew.Chem., 100 (1988) 970; Angew. Chem. Int. Ed. Engl., 27 (1988) 939.

4 Dies wurde anhand einer Kristallstrukturanalyse von vermeintlichem $2 \mathrm{~b}$ gefunden. K. Sünkel, unveröffentlicht.

5 (a) M.D. Rausch, J. Org. Chem., 26 (1961) 3579; (b) M. Herberhold und P. Leitner, J. Organomet. Chem., 336 (1987) 153.

6 (a) G. Boche, N. Mayer, M. Bernheim und K. Wagner, Angew. Chem., 90 (1978) 733; Angew. Chem. Int. Ed. Engl., 17 (1978) 687; (b) F.D. King und D.R.M. Walton, Synthesis, (1975) 788.

7 M. Sato, T. Ito, I. Motoyama, K. Watanabe und K. Hata, Bull. Chem. Soc. Jpn., 42 (1969) 1976.

8 Die Struktur dieser polymeren $\mathrm{Cu}^{1}-\mathrm{Cu}^{11}$-Verbindung konnte kristallographisch bestimmt werden: $\mathbf{K}$. Sünkel, unveröffentlicht.

9 C. Grundmann in J. Falbe (Htsg.), Houben-Weyl, Methoden der Org.Chem., 4. Aufl., Bd. E5, S. 1358, G. Thieme Verlag, Stuttgart, New York, 1985.

10 Kristalldaten: Größe: $0.26 \times 0.17 \times 0.52 \mathrm{~mm}^{3}$; monoklin $C 2 / c ; a$ 1538.3(4), $b$ 2037.1(12), $c 953.85(34)$ pm, $\beta 113.602(25)^{\circ} . V 2.738 \mathrm{~nm}^{3} . Z=8.3133$ Reflexe gemessen $\left(4 \leq 2 \theta \leq 45^{\circ} ; \pm h,+k,+l\right), 1625$ unabhängig, 1313 davon beobachtet mit $(I \geq 2 \sigma(I))$. Syntex $\mathrm{P} 2_{1}$-Diffraktometer, Mo- $K_{\alpha}$-Strahlung, Graphitmonochromator, $\omega$-Scan $\left(\Delta \omega=1^{\circ}, 4 \leq \dot{\omega} 30^{\circ} / \mathrm{min}\right)$. Empirische Absorptionskorrektur mit 10 Reflexen: $T_{\max }=0.442, T_{\min }=0.389, \mu\left(\mathrm{Mo}-K_{\alpha}\right)=17.10 \mathrm{~cm}^{-1}$. Strukturlösung mit SHELXTL 4.1 mit Hilfe direkter Methoden und anschließender Differenzfouriercyclen. 112 verfeinerte Parameter (Mangan, Chlor, Stickstoff und Acylsauerstoff anisotrop; Wasserstoffe am Stickstoff nicht eindeutig lokalisierbar und deshalb weggelassen). $R=0.0599, R_{\mathrm{w}}=0.0642$. Restelektronendichte 0.7 , lokalisiert in der Anisotropie der Metallcarbonylgruppen.

Ebenengleichungen:

E1 (C11 bis C15): $2.183 x+15.018 y-6.316 z+0.2142=0$

E2 (C11, C16, O4, N1) $10.273 x-15.129 y-2.98 z-0.5045=0$

Atomabstände zu EI (in pm):

Mn1: 176.7; C11: 1.49; C12: - 1.21; C13: 0.46; C14:0.50; C15: -1.24; C16: 1.18; O4: 97.76; N1: -105.36 ; Cl12: -12.40 ; C113 - 15.53; Cl14: -16.69 ; Cl15: -17.81 .

Weitere Einzelheiten zur Kristallstrukturuntersuchung können beim Fachinformationszentrum Energie, Physik, Mathematik GmbH, D-7514 Eggenstein-Leopoldshafen 2, unter Angabe der Hinterlegungsnummer CSD-53639, der Autoren und des Zeitschriftenzitats angefordert werden.

11 K.J. Reimer und A. Shaver, Inorg. Chem., 14 (1975) 2707. 